\title{
Steady analysis of transcritical flows in collapsible tubes with discontinuous mechanical properties: implications for arteries and veins
}

\author{
A. Siviglia ${ }^{1} \dagger$ and M. Toffolon ${ }^{2}$ \\ ${ }^{1}$ Laboratory of Hydraulics, Hydrology and Glaciology VAW, ETH Zurich, Switzerland \\ ${ }^{2}$ Department of Civil, Environmental and Mechanical Engineering, University of Trento, Italy
}

(Received 18 February 2013; revised 4 October 2013; accepted 9 October 2013; first published online 4 November 2013)

We study the conditions under which discontinuous mechanical properties of a collapsible tube can induce transcritical flows, i.e. the transition through the critical state where the speed index (analogous to the Mach or the Froude numbers for compressible and free surface flows, respectively) is one. Such a critical transition may strongly modify the flow properties, cause a significant reduction in the crosssectional area of the tube, and limit the flow. General relationships are obtained for a short segment using a one-dimensional model under steady flow conditions. Marginal curves delimiting the transcritical regions are identified in terms of the speed index and the cross-sectional area ratio. Since there are many examples of such flows in physiology and medicine, we also analyse the specific case of prosthesis (graft or stent) implantation in blood vessels. We then compute transcritical conditions for the case of stiffness and reference area variations, considering a collapsible tube characterized by physiological parameters representative of both arteries and veins. The results suggest that variations in mechanical properties may induce transcritical flow in veins but is unrealistic in arteries.

Key words: biological fluid dynamics, blood flow, flow-vessel interactions

\section{Introduction}

Most physiological flows are characterized by the transport of fluids through flexible tubes. Prominent examples are air flow in the airways, urine flow in the ureter and urethra, and blood flow in arteries and veins (Grotberg \& Jensen 2004). In this context the fluid-structure interaction plays an important role (Heil \& Hazel 2011). In fact, interactions between the pressure of the fluid and compliant walls determine the velocity profiles and wall displacement patterns. The equations governing the fluid-structure interaction problem in the one-dimensional case are the continuity and momentum equations and a tube law closure which describes how the pressure changes with the cross-sectional area. A detailed analysis of the solutions of these governing equations under steady conditions, assuming incompressible and inviscid flows, has been developed by Shapiro (1977). He described the solutions in terms of the dimensionless parameter speed index, $S$, which controls the flow conditions, 
showing that different patterns are possible for flows which are subcritical $(S<1)$ rather than supercritical $(S>1)$. Moreover, he showed that smooth or abrupt transitions through critical conditions $(S=1)$ along the tube are possible under particular geometrical and mechanical conditions (e.g. cross-sectional area restriction). Shapiro also describes some peculiarity of collapsible-tube flows when $S=1$, namely (i) choking, and (ii) elastic jumps. In the first case a smooth transition connects a subcritical to a supercritical flow and a flow limitation occurs: given the conditions upstream of the critical point (where $S=1$ ), there is a maximum steady flow rate that the tube can convey independently of the difference between upstream and downstream pressures. In the second case, a rapid transition from supercritical to subcritical flow occurs, imposing a rapid change of cross-sectional area, and this is the only mechanism of significant energy dissipation in the inviscid solution.

Steady-state analysis has been widely employed for investigating problems relevant from a physiological point of view. Among them, a detailed study of choking and elastic jumps occurring through stenotic arteries is given in $\mathrm{Ku}$, Zeigler \& Downing (1990), and an analysis of choking phenomena in a lung-like model has been carried out by Elad, Kamm \& Shapiro (1987). The behaviour of blood pressure and flow rate in the jugular vein of the giraffe has been investigated by Pedley, Brook \& Seymour (1996), while a mathematical simulation of forced expiration is performed in Elad, Kamm \& Shapiro (1988), and an analysis of forced expiration under steady compressible flow is provided in Elad, Kamm \& Shapiro (1989). The study of steady flows and of the threshold of stability giving oscillatory states has been analysed in Cancelli \& Pedley (1985) and Jensen \& Pedley (1989). Numerical unsteady calculations to study the influence of different physiological parameters on a forced expiration are given in Elad \& Kamm (1989).

The aim of this work is to present an analytical approach for determining when a fluid flowing inside a compliant tube can pass through critical conditions due to mechanical discontinuous variations. Therefore we analyse the conditions under which flow limitations (choking) and elastic jumps can occur. The approach we use is similar to the one proposed by Marchi (1968) for determining when the reduction in width due to the presence of bridge piers in rivers can induce transition through critical conditions. The approach resembles, while being more general, that proposed by Pedley (2000) for studying the effects of a non-uniform external pressure applied to a uniform collapsible tube. We perform a one-dimensional analysis under steady flow conditions, assuming that the mechanical variations occur in such a short distance that friction plays a negligible role and the total pressure stays reasonably constant.

We are motivated by situations of medical interest since flow limitations and elastic jumps can have adverse physiological effects. Our analysis is focused on physical situations in which certain mechanical properties that characterize the tube material change rapidly, or even discontinuously. Physical quantities of interest are vessel wall thickness and Young's modulus. Prominent examples arise in the surgical treatment of abdominal aortic aneurysms (AAA) (e.g. Sakalihasan, Limet \& Defawe 2005). In this case a localized dilatation is present in the aorta. When it grows over a certain limit the risk of rupture becomes high. With a surgical procedure vascular repair is obtained by using prosthesis (grafts) or by inserting stents. Stents are also implanted in veins in different circumstances. For example, they are used in the treatment of chronic cerebrospinal venous insufficiency (CCSVI) (e.g. Zamboni et al. 2009), in which occlusion of some veins (e.g. the jugular vein) can alter the normal blood flux resulting in several pathological effects. In this case stents are used to hold the vein open. Since the material used for making these repairs is very different from that 
of the hosting vessel, there is a potential difference in terms of Young's moduli and wall thickness. Associated with this, the repair can also have a diameter that can be slightly different (higher or smaller) than that of the receiving vessel (e.g. Canić et al. 2005). Such a mismatch between the mechanical prosthesis and the natural vessel may cause disturbances that can have several physiological implications. For example, aortic grafts may increase the arterial pulse wave velocity (Lantelme et al. 2009; Kadoglou et al. 2012) and pressure wave reflection can occur just upstream of the implantation region (Charonko, Ragab \& Vlachos 2009).

Mathematical analyses of the one-dimensional unsteady problem with variable mechanical properties are also available in the literature. For example, numerical investigations of the variation of the pulse wave velocity have been performed by Vardoulis et al. (2011), alteration in the pressure pattern in the case of prosthesis implantation in large arteries has been analysed by Sherwin et al. (2003), while the possibility of stent migration at the anchoring sites, which can lead to the failure of the repair, has been investigated by Canić et al. (2005). Recently, Toro \& Siviglia (2013) proposed a new mathematical approach to flow in collapsible tubes dealing with discontinuous mechanical properties, and Müller, Parés \& Toro (2013) provided a numerical solution.

The structure of the paper is as follows. In $\S 2$ we describe the one-dimensional mathematical model, the closure relationships and the simplified equations under steady conditions. In $\S 3$ we present the results that analyse the effects of variations in geometrical and mechanical properties on the flow in a collapsible tube, while in $\S 4$ we discuss the implications of variations in mechanical properties for the occurrence of critical conditions in arteries and veins. Conclusions are drawn in $\S 5$.

\section{Mathematical model and assumptions}

\subsection{One-dimensional governing equations}

The basic equations for one-dimensional flow in collapsible tubes are obtained from the balances of mass and momentum for the fluid phase. Assuming a constant density $\rho$ for the fluid and neglecting gravity, the governing equations read as

$$
\begin{gathered}
\frac{\partial A}{\partial t}+\frac{\partial Q}{\partial x}=0, \\
\frac{\partial Q}{\partial t}+\frac{\partial(u Q)}{\partial x}+\frac{A}{\rho} \frac{\partial p}{\partial x}=-R u,
\end{gathered}
$$

where $A$ is the cross-sectional area of the tube, $u$ is the averaged velocity of the fluid and $p$ its pressure, $Q=u A$ is the flow rate, and $R$ is the flow resistance per unit length of the tube, assumed to be a known function of the unknowns.

Since only two partial differential equations, (2.1) and (2.2), are provided for three unknowns (namely $A, u$ and $p$ ), an additional relation is required to complete the system. This is provided by the tube law, which relates the pressure $p$ to the wall displacement via the cross-sectional area $A$ coupling the elastic properties of the vessel to the fluid dynamics. The main purpose of law (2.3) is to capture the tube's flexibility at small negative pressures as collapse is initiated, whilst maintaining the properties of a stiffening response for higher negative or positive pressures. In this work we assume (Elad et al. 1987)

$$
\psi(\alpha, K)=p-p_{e}=K\left(\alpha^{\beta_{1}}-\alpha^{-\beta_{2}}\right),
$$


where the transmural pressure $\psi$ is the difference between the internal pressure $p$ and the external pressure $p_{e}$. Here $K$ is a quantity that represents the elastic properties of the material and the wall thickness, and is called the effective stiffness of the tube (see Shapiro 1977), and $\alpha=A / A_{0}$ is the area ratio, where $A_{0}$ represents the undistorted cross-sectional area, at zero transmural pressure $(\psi=0)$, while the two exponents $\beta_{1}$ and $\beta_{2}$ are real numbers. We restrict our analysis to the range $\beta_{1} \geqslant 0$ and $0<\beta_{2} \leqslant 2$, which are the usual ranges used for describing the behaviour of collapsible tubes and in which system of equations (2.1) and (2.2) is hyperbolic (Toro \& Siviglia 2013). If $\alpha>1$ the transmural pressure is positive and the tube is inflated, while if $\alpha<1$ the transmural pressure is negative and the tube is collapsed.

The tube law determines the wave speed $c$ of small area perturbations, which in this case is given by (Toro \& Siviglia 2013)

$$
c=\sqrt{\frac{A \mathrm{~d} \psi}{\rho} \frac{\mathrm{d} A}{\mathrm{~d} A}}=\sqrt{\frac{K}{\rho}\left(\beta_{1} \alpha^{\beta_{1}}+\beta_{2} \alpha^{-\beta_{2}}\right)} .
$$

Following Shapiro (1977), we introduce the speed index $S=u / c$, a dimensionless controlling parameter that is the analogous to the Mach number for compressible flows and the Froude number for open channel flows. By substituting (2.4) it follows that

$$
S^{2}=\frac{\rho u^{2}}{\alpha \frac{\mathrm{d} \psi}{\mathrm{d} \alpha}}=\frac{1}{\chi \alpha^{2}\left(\beta_{1} \alpha^{\beta_{1}}+\beta_{2} \alpha^{-\beta_{2}}\right)},
$$

where the dimensionless parameter

$$
\chi=\frac{K}{\rho u_{0}^{2}}
$$

characterizes the flow at zero transmural pressure $\left(u_{0}=Q / A_{0}\right)$. It is worth noticing that this parameter is the square of a particular speed index which is the ratio of the wave speed $\sqrt{K / \rho}$ and the velocity $u_{0}$.

It will prove useful in the following sections to introduce a particular value of the parameter $\chi$,

$$
\chi_{c e}=\frac{1}{\beta_{1}+\beta_{2}},
$$

obtained from (2.5) for the occurrence of critical conditions $(S=1)$ in flows with equilibrium cross-sectional area $\left(A=A_{0}, \alpha=1\right)$. In the range for $\beta_{1}$ and $\beta_{2}$ considered in this paper, the value $\chi_{c e}$, as suggested by relation (2.5), discriminates flows having critical conditions for a positive transmural pressure $\left(\alpha>1, \chi<\chi_{c e}\right)$ from those that are critical for a negative transmural pressure $\left(\alpha<1, \chi>\chi_{c e}\right)$.

\subsection{Steady problem}

Let us consider a short segment of a collapsible tube under steady $(\partial / \partial t \equiv 0)$ conditions. Equations (2.1) and (2.2) can be simplified as follows:

$$
\begin{gathered}
\frac{\mathrm{d} Q}{\mathrm{~d} x}=0, \\
\frac{\mathrm{d}\left(u^{2} A\right)}{\mathrm{d} x}+\frac{A}{\rho} \frac{\mathrm{d} p}{\mathrm{~d} x}=-R u .
\end{gathered}
$$




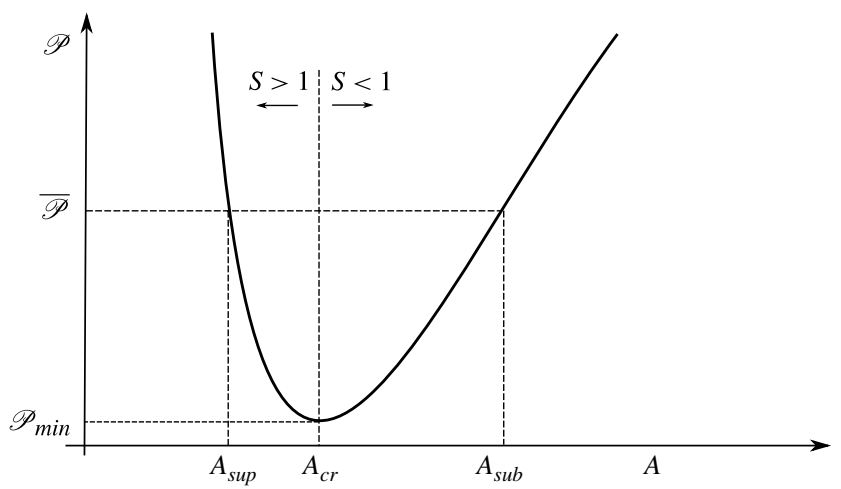

FIGURE 1. Plot of the total pressure as a function of the cross-sectional area for assigned $Q$, $K, p_{e}$ and $A_{0}$.

Equation (2.8) states that the flow rate remains constant along the tube, while (2.9) reduces to

$$
\frac{\mathrm{d}}{\mathrm{d} x}\left(\frac{u^{2}}{2}+\frac{p}{\rho}\right)=-\frac{R u}{A} .
$$

If we also consider inviscid $(R=0)$ conditions, this equation states that the total pressure (or stagnation pressure)

$$
\mathscr{P}=\rho \frac{Q^{2}}{2 A^{2}}+\psi\left(\frac{A}{A_{0}}, K\right)+p_{e}
$$

remains constant along the tube. Since the variation of $\mathscr{P}$ is given by the integral of the right-hand side of (2.10) along a tube segment, this assumption remains valid for a short distance for viscous flows too.

Figure 1 shows the behaviour of the function $\mathscr{P}=\mathscr{P}(A)$ for fixed values of $Q, K$, $p_{e}$ and $A_{0}$. For a given total pressure $\overline{\mathscr{P}}>\mathscr{P}_{\text {min }}$, two different values of cross-sectional area are physically possible (see figure 1 ), namely $A_{\text {sub }}$ and $A_{\text {sup }}$, which lie on the right and left side of the critical value $A_{c r}$, respectively. Thus, $A_{c r}$ is the value for which the total pressure is minimum, and can be computed on imposing $\left.(\mathrm{d} \mathscr{P} / \mathrm{d} A)\right|_{Q, K, A_{0}}=0$.

\section{Geometrical and mechanical tube variations}

In this section we investigate the effects of a variation of the geometrical characteristics (i.e. the cross-sectional area at equilibrium $A_{0}$ ) and mechanical properties of the tube wall (i.e. the stiffness parameter $K$ ) on the flow in a collapsible tube. We identify with the subscript $m\left(A_{0 m}, K_{m}, \mathscr{P}_{m}\right)$ the values of the parameters in a modified region (see figure 2), whose length is assumed to be short enough to neglect the effect of friction and to enforce that $\mathscr{P}_{m}=\mathscr{P}$ across the discontinuity. We also neglect the total pressure losses due to sudden enlargements; in any case, their consideration does not qualitatively change the overall description of the process and the conclusions drawn at the end of this paper.

Among all the possible variations, we are interested in those geometrical and mechanical changes that can lead to transcritical flows. As an example we show how a subcritical and a supercritical flow can approach critical conditions due to a sudden 


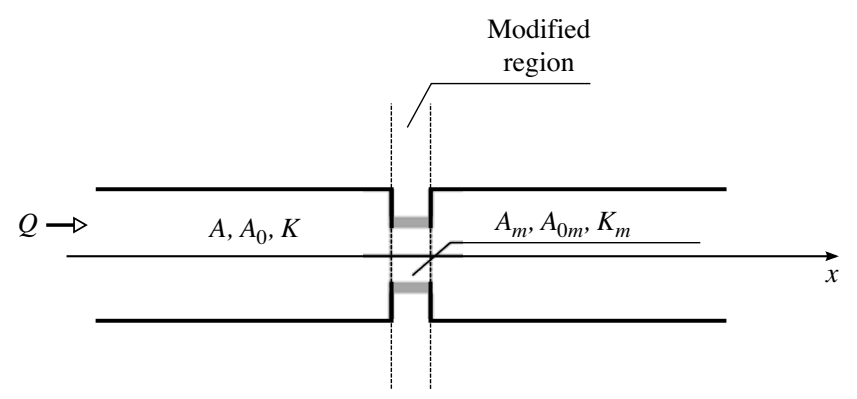

FIGURE 2. Sketch of the considered modified configuration.

restriction of the undistorted cross-sectional area $A_{0}$. We solve (2.10), which governs the steady-state problem, following the procedure described in the Appendix. We consider two different situations: (i) a subcritical and (ii) a supercritical flow passing through a stenosed collapsible tube (i.e. $A_{0 m} / A_{0}<1$ ). The situation is illustrated in figure 3, where for the sake of clarity a sinusoidal stenosis is used instead of the abrupt variation sketched in figure 2. The figure shows the longitudinal variation of the speed index $S$ (figure $3 e$ ), the velocity $u$ (figure $3 f$ ), the cross-sectional area $A$ (figure $3 c$ ), the transmural pressure $\psi$ (figure $3 d$ ), and the area ratio $\alpha$ (figure $3 b$ ) as a function of the position $\xi=x / D$, with $D$ the nominal tube diameter. The flow is inviscid $(\mu=0)$ and from left to right. It is seen that a subcritical flow, while approaching the stenosis, decreases its cross-sectional area, becomes faster and thus increases its speed index. In contrast, a supercritical flow increases its cross-sectional area and becomes slower, with a decrease in the speed index. Hence, both subcritical and supercritical flows move towards the critical condition in the stenosis, and could possibly become transcritical and change its nature as the degree of the stenosis is more severe.

It is the aim of this paper to understand when a mechanical variation of the vessel properties is able to induce critical flow conditions. We are interested in deriving the marginal curves for the transcritical flow, namely the condition that causes a critical flow in the modified region, i.e. $S_{m}=1$ and $A_{m}=A_{c r}$. In this respect, our approach is similar to the one proposed by Marchi (1968) for the analysis of cross-section narrowing in rivers. In the next two sections we consider the transcritical conditions for a simplified and the complete version of the tube law.

\subsection{Simplified tube law}

A simplified version of the tube law is obtained by imposing $\beta_{2}=0$ in the relation (2.3). This is normally used in a physiological context to describe flows in arteries (e.g. Canić 2002; Sherwin et al. 2003; Alastruey et al. 2007; Liang et al. 2009). The use of such a simplified law allows us to derive an expression for the critical area in closed form, which reads

$$
A_{c r}=\left(\frac{\rho Q^{2} A_{0}^{\beta_{1}}}{K \beta_{1}}\right)^{1 /\left(2+\beta_{1}\right)}=\frac{A_{0}}{\left(\chi \beta_{1}\right)^{1 /\left(2+\beta_{1}\right)}} .
$$

Definition (3.1) is obtained by imposing $S=1$ in (2.5), so $A_{c r}$ discriminates subcritical flows $(S<1)$ from supercritical flows $(S>1)$. Two different values of cross-sectional 
(a)

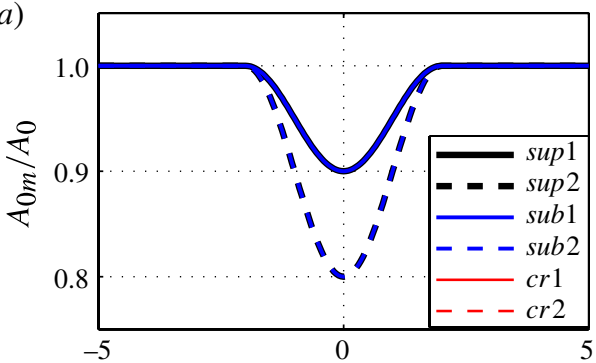

(c)

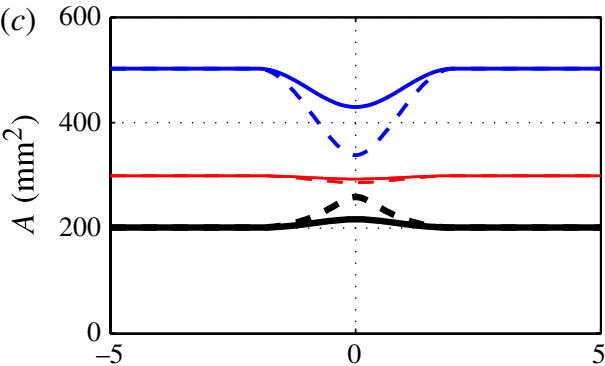

(e)

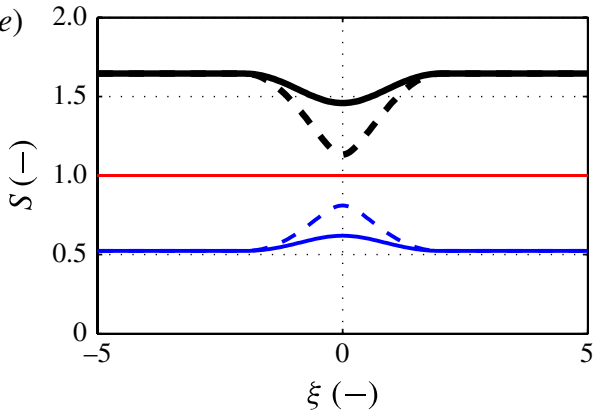

(b)

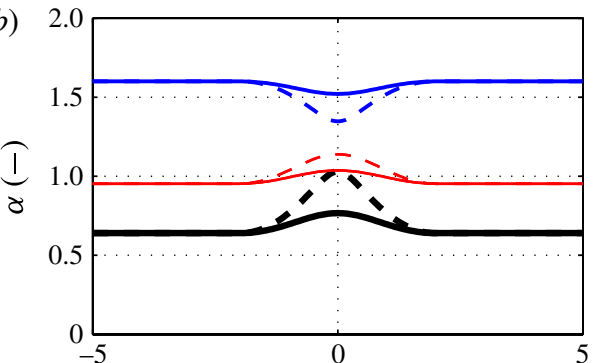

(d)

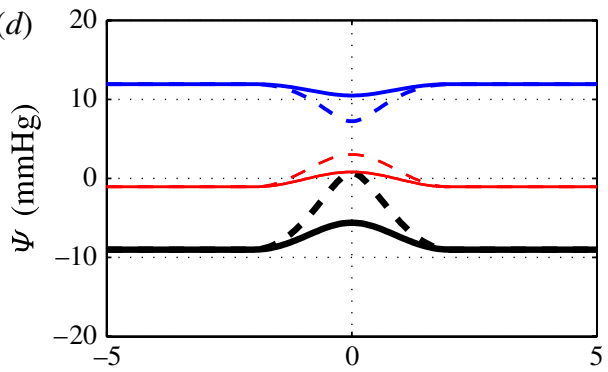

(f)

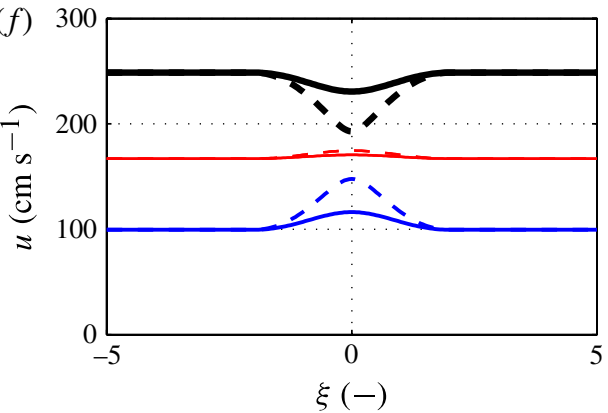

FIGURE 3. (Colour online) Steady profiles for an inviscid flow $(R=0)$ through a stenosis in a collapsible tube $\left(\beta_{1}=1 / 2, \beta_{2}=0 ; \chi_{c e}=2\right)$ as a function of the dimensionless distance $\xi=x / D$ ( $D$ being the tube diameter): reference area ratio $A_{0 m} / A_{0}(a)$, cross-sectional area $A$ $(c)$, speed index $S(e)$, area ratio $\alpha(b)$. Transmural pressure $\psi(d)$, flow velocity $u(f)$. The subcritical case $(S=0.52$, blue line; $\psi=11.9 \mathrm{mmHg}$ as downstream boundary condition) is compared with the supercritical case $(S=1.64$, black thicker line; $\psi=-9 \mathrm{mmHg}$ as upstream boundary condition) for two different stenoses characterized by a sinusoidal variation (maximum restriction: $A_{0 m} / A_{0}=0.9$, solid line; $A_{0 m} / A_{0}=0.8$, dashed line). Critical values of the variables are drawn with thin red lines. Other data: density $\rho=1050 \mathrm{~kg} \mathrm{~m}^{-3}$, discharge $Q=500 \mathrm{ml} \mathrm{s}^{-1}$, tube radius $R_{0}=1 \mathrm{~cm}$, stiffness $K=6000 \mathrm{~Pa}$, resulting in a value of the parameter $\chi=2.26$.

area are mathematically possible for a given value of total pressure $\overline{\mathscr{P}}>\mathscr{P}_{\text {min }}$ (see figure 1): one subcritical $\left(A>A_{c r}\right)$ and one supercritical $\left(A<A_{c r}\right)$.

Using the definitions of the speed index and the area ratio $\alpha$, the total pressure (2.11) can be expressed as

$$
\mathscr{P}=K \alpha^{\beta_{1}}\left(\frac{\beta_{1}}{2} S^{2}+1\right)+p_{e}-K .
$$


Then, considering inviscid flow, assuming constant $p_{e}$, imposing $\mathscr{P}=\mathscr{P}_{m}$ and the critical conditions in the modified region $m$, i.e. $\mathscr{P}_{m}=\mathscr{P}\left(S=1 ; A=A_{c r}\right)$, we obtain

$$
K \alpha^{\beta_{1}}\left(\frac{\beta_{1}}{2} S^{2}+1\right)-K=K_{m} \alpha_{m}^{\beta_{1}}\left(\frac{\beta_{1}}{2}+1\right)-K_{m},
$$

where $\alpha_{m}=A_{c r} / A_{0 m}$ and $S^{2}=\left(\chi \beta_{1} \alpha^{2+\beta_{1}}\right)^{-1}$ from (2.5). Equation (3.3) can be manipulated to obtain the following relationships, expressed in terms of $S$ and $\alpha$, respectively:

$$
\begin{aligned}
& \left(\frac{\beta_{1} S^{2}}{2}+1\right) S^{-2 \beta_{1} /\left(2+\beta_{1}\right)} \\
& =\left(1-\frac{K_{m}}{K}\right)\left(\beta_{1} \chi\right)^{\beta_{1} /\left(2+\beta_{1}\right)}+\left(\frac{\beta_{1}}{2}+1\right)\left(\frac{A_{0 m}}{A_{0}}\right)^{-2 \beta_{1} /\left(2+\beta_{1}\right)}\left(\frac{K_{m}}{K}\right)^{2 /\left(2+\beta_{1}\right)}, \\
& \left(\frac{1}{2 \chi \alpha^{2+\beta_{1}}}+1\right) \alpha^{\beta_{1}} \\
& =\left(1-\frac{K_{m}}{K}\right)+\left(\frac{\beta_{1}}{2}+1\right)\left(\frac{A_{0 m}}{A_{0}}\right)^{-2 \beta_{1} /\left(2+\beta_{1}\right)}\left(\frac{K_{m}}{K}\right)^{2 /\left(2+\beta_{1}\right)}\left(\beta_{1} \chi\right)^{-\beta_{1} /\left(2+\beta_{1}\right)}
\end{aligned}
$$

These relationships are equivalent and can be used to determine the marginal transcritical conditions provided that the geometrical and mechanical characteristics of the tube $\left(A_{0}, A_{0 m}, K, K_{m}, \beta_{1}\right)$ are known.

\subsubsection{Geometrical variations $\left(A_{0 m} \neq A_{0}, K_{m}=K\right)$}

In this section we consider the case of geometrical variations only (i.e. $K_{m} / K=1$ ), so that the first term on the right-hand side of (3.4) vanishes. Thus the dependence on $\chi$ disappears and only one curve is detectable in the plane $\left(S-A_{0 m} / A_{0}\right)$ (see figure $4 a$ ). On the other hand, (3.5) provides different marginal curves in the plane $\left(\alpha-A_{0 m} / A_{0}\right)$ for different values of $\chi$ (figure $4 b$ ).

If the reference area of the tube widens in the modified region $\left(A_{0 m} / A_{0}>1\right)$, (3.4) does not have solutions for any value of $S$, implying that no transition can occur. For a stenosis $\left(A_{0 m} / A_{0}<1\right)$, instead, (3.4) has two solutions for the speed index $S$ : the two roots are the speed index limits $S_{L}^{\mathrm{I}}<1$ and $S_{L}^{\mathrm{II}}>1$ (see figure $4 a$ ). An undisturbed subcritical flow remains subcritical when it passes through the modified region if $S<S_{L}^{\mathrm{I}}$ (see example in figure 3), and analogously an undisturbed supercritical flow remains supercritical if $S>S_{L}^{\text {II }}$ (see example in figure 3). The region in between, below the marginal curve, represents the transcritical flow area: here, the flow changes its nature, passing from subcritical to supercritical through the critical conditions. The asymmetric shape of the transcritical curve also suggests that supercritical flows are more likely to achieve critical conditions than subcritical flows. In general, the stronger the cross-section narrowing (smaller $A_{0 m} / A_{0}$ ), the wider the range of $S$ for which a transcritical flow may occur.

Considering the solution of (3.5) in the plane $\left(\alpha-A_{0 m} / A_{0}\right)$, we obtain different transcritical curves for different values of $\chi$ (see figure $4 b$ ). Also in this case, for given values of the ratio $A_{0 m} / A_{0}$ and $\chi$ we have that two limit values for the area ratio exist, namely $\alpha_{L}^{I}$ and $\alpha_{L}^{\mathrm{II}}$, corresponding to $S_{L}^{\mathrm{I}}<1$ and $S_{L}^{\mathrm{II}}>1$, respectively: if $\alpha_{L}^{\mathrm{II}}<\alpha<\alpha_{L}^{\mathrm{I}}$ the incoming flow passes through critical conditions. However, as suggested by relation (3.1), the transcritical region shifts from lower values of $\alpha$ for large $\chi$ to higher values of $\alpha$ for small $\chi$, being centred on $\alpha=1$ for $\chi=\chi_{c e}=\beta_{1}^{-1}$. This observation suggests that transcritical flows occur more easily for values 

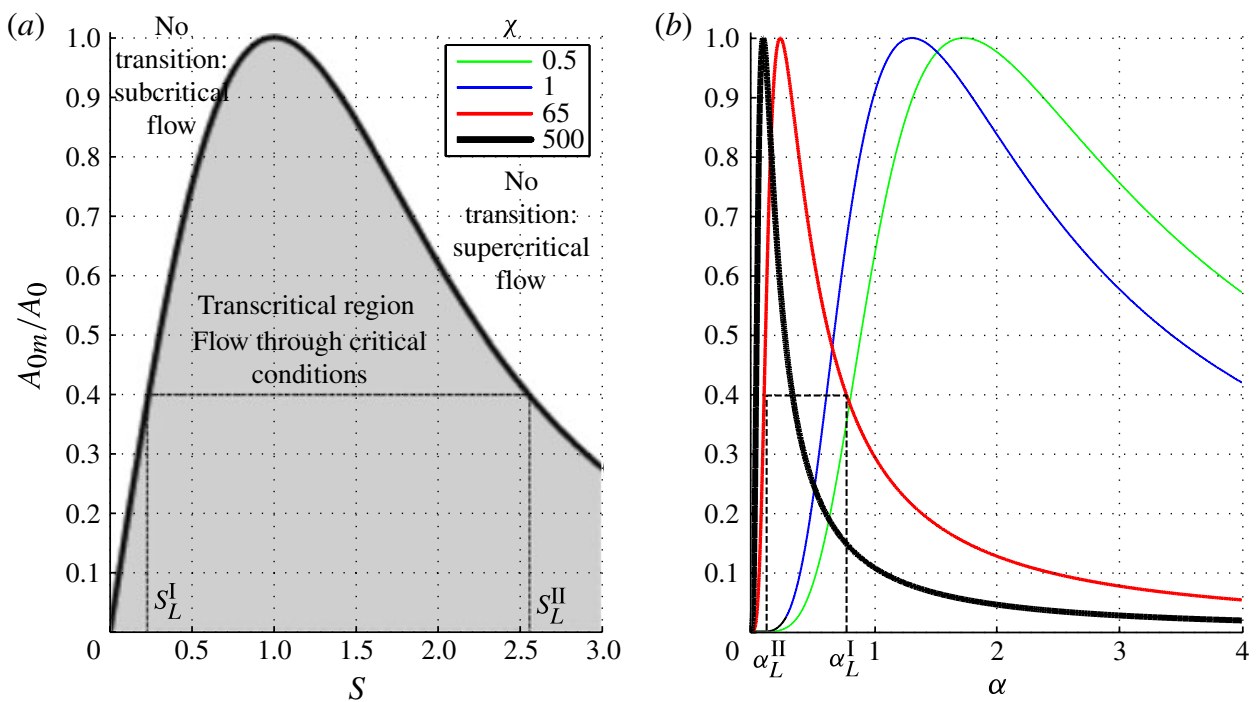

FIGURE 4. (Colour online) Geometrical variations: transcritical curves in the plane $(S$ $\left.A_{0 m} / A_{0}\right)(a)$ and $\left(\alpha-A_{0 m} / A_{0}\right)(b)$ for different values of $\chi$, in the case of simplified tube law $\left(\beta_{1}=1 / 2, \beta_{2}=0, \chi_{c e}=2\right)$ and constant mechanical properties $\left(K_{m} / K=1\right)$.

of $\chi \sim \chi_{c e}$, since in this range the transmural pressure $\psi(\alpha)$ is not required to be too large (as it would be for $\alpha \gg 1$ ) or too small $(\alpha \ll 1)$.

In order to improve the description of the phenomenon, in figure 5 we explicitly show the flow pattern occurring during the transition through critical conditions because of a restriction. For this sake, we solve the governing equation (2.10) as described in the Appendix, also taking into account the friction term for a more complete analysis. In this example, which refers to the same flow data as the subcritical case in figure 3 , the stenosis $A_{0 m} / A_{0}=0.5$ produces a transition and divides the domain into two regions. Upstream the flow is subcritical, while downstream it becomes supercritical until, eventually, the downstream boundary condition causes a further transition to a subcritical flow through an elastic jump. This latter passage is visible (at $\xi=15.9$ ) only when viscous dissipation is accounted for, while when $R=0$ (and $A_{0}$ and $K$ are also constant) the cross-sectional area profiles are straight lines like the ones depicted in figure 3. We note that the undisturbed subcritical flow is characterized by a speed index $S=0.52$, which lies in the transcritical region delimited in figure 4 . Moreover, the two limit values $S_{L}^{\mathrm{I}}=0.30$ and $S_{L}^{\mathrm{II}}=2.27$ correctly identify the flow conditions just upstream and downstream of the transition, respectively.

\subsubsection{Variations in mechanical properties $\left(A_{0 m}=A_{0}\right.$ and $\left.K_{m} \neq K\right)$}

Unlike the previous case, when $K_{m} \neq K$ the dependence on $\chi$ does not vanish in (3.4). Therefore, marginal curves are plotted in figure 6 for different values of $\chi$ both in the $\left(S-K_{m} / K\right)$ plane (figure $\left.6 a, c\right)$ and in the $\left(\alpha-K_{m} / K\right)$ plane (figure $\left.6 b, d\right)$. Moreover, since the behaviour changes for $\chi \gtrless \chi_{c e}$, we separate the two cases in figure $6(a, b)$ and figure $6(c, d)$. As in the previous case, two limit values for $S$ and $\alpha$ can be identified for a given stiffness ratio $K_{m} / K$, and the flow is transcritical if the unmodified conditions lie within the region delimited by the marginal curves. 
(a)

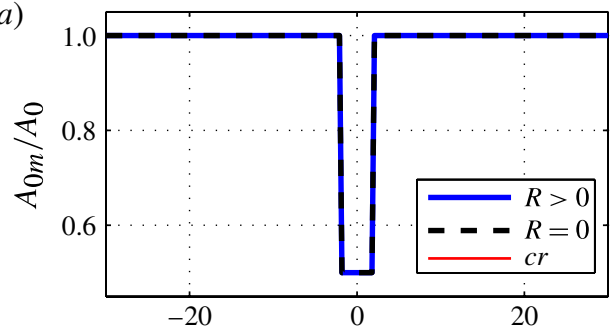

(c) 1000

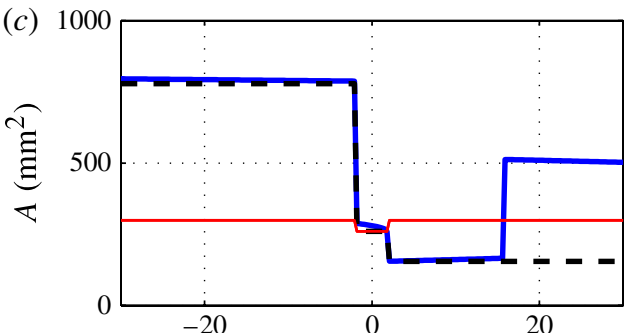

$(e)$

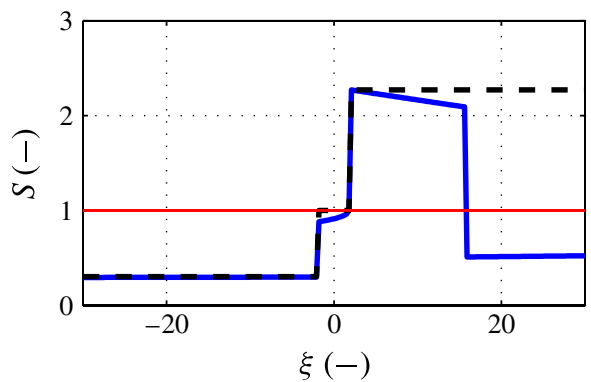

(b)

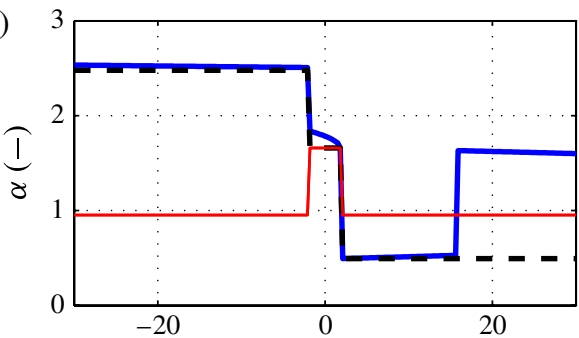

(d)
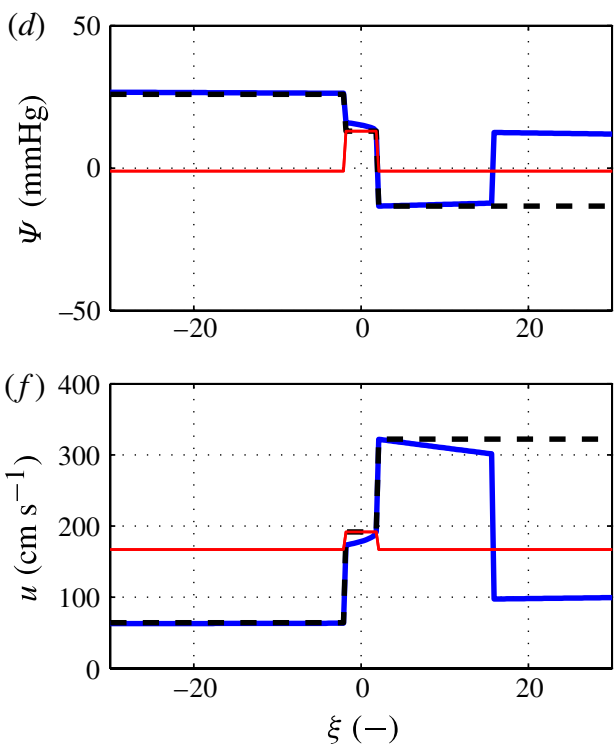

FIgURE 5. (Colour online) Steady profiles with transcritical conditions through a stenosis $\left(A_{0 m} / A_{0}=0.5\right)$ for an inviscid $(R=0$, black dashed line $)$ and a viscous $(R>0, \mu=$ $4.0 \times 10^{-3} \mathrm{~Pa} \mathrm{~s}$, blue solid line) flow. The reference undisturbed flow is subcritical $(S=0.52)$ with $\psi=11.9 \mathrm{mmHg}\left(A=503 \mathrm{~mm}^{2}\right)$ as downstream boundary condition. The panels and other data are as in figure 3.

As is implied by the discussion of the definition (2.5), the transcritical conditions occur for $\alpha>1$ (positive transmural pressure) when $\chi<\chi_{c e}$, and vice versa (see figure $6 b, d)$. Interestingly, $\chi \gtrless \chi_{c e}$ also distinguishes two contrasting cases for the onset of transcritical flows: when $\chi<\chi_{c e}$ only an increase in the stiffness in the modified region $\left(K_{m} / K>1\right)$ is able to produce critical conditions, while the marginal curves are in the region of lower stiffness $\left(K_{m} / K<1\right)$ for $\chi>\chi_{c e}$. Finally, the transcritical region is restricted to a single point $\left(\alpha=1, S=1, K_{m} / K=1\right)$ for $\chi=\chi_{c e}$, i.e. the flow is critical in the modified region only if the approaching flow is already critical at the equilibrium area $A_{0}$.

\subsubsection{Variations in geometrical and mechanical properties $\left(A_{0 m} \neq A_{0}\right.$ and $\left.K_{m} \neq K\right)$}

This is the general case in which variations of both the equilibrium area $A_{0}$ and the stiffness parameter $K$ are considered. In figure $7(a, b)$ we plot the transcritical curves in the $\left(S-K_{m} / K\right)$ plane and in the $\left(\alpha-K_{m} / K\right)$ plane for different values of the parameter $\chi$ and for a fixed ratio $A_{0 m} / A_{0}=0.75$. This means that we are considering the case in which the stiffness parameter changes and the cross-sectional area at the equilibrium is reduced in the modified region. In this case the differences from the case of pure 


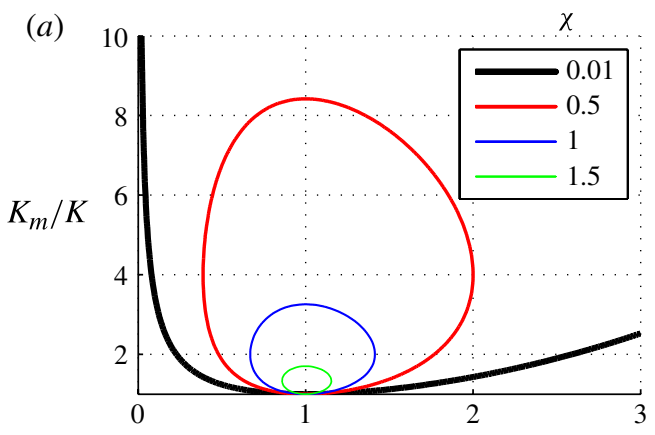

(b)
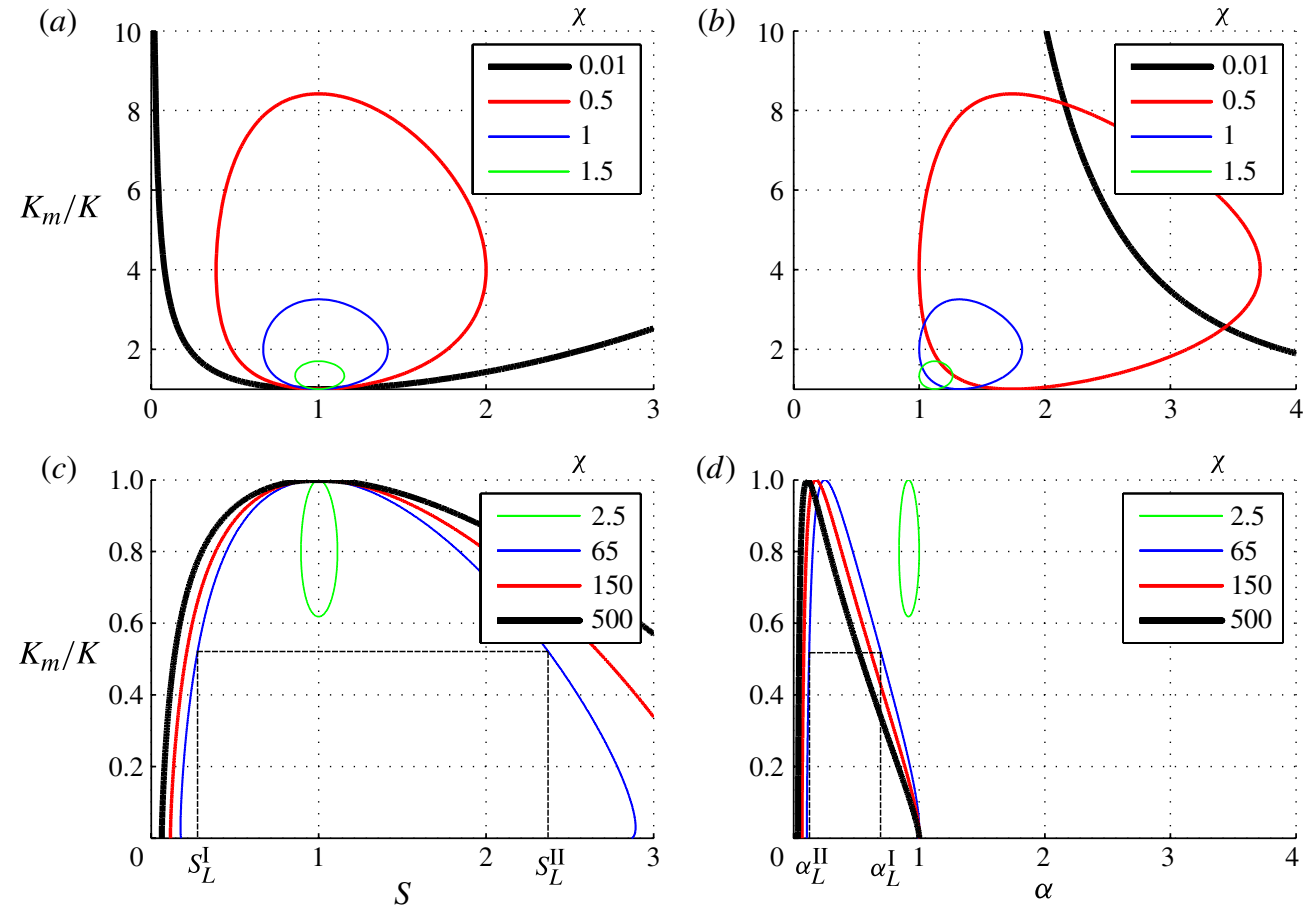

(d)

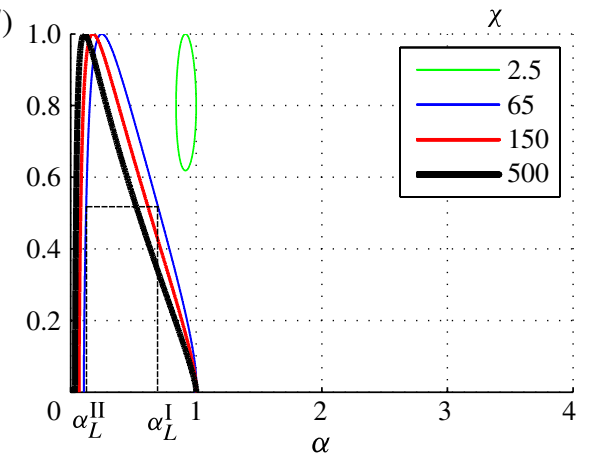

FIGURE 6. (Colour online) Mechanical variations: transcritical conditions of the ratio $K_{m} / K$ as a function of the speed index $S(a, c)$ and of the area ratio $\alpha(b, d)$, for different values of $\chi$. Results are obtained for the simplified tube law $\left(\beta_{1}=1 / 2, \beta_{2}=0, \chi_{c e}=2\right)$ and constant area $\left(A_{0 m} / A_{0}=1\right)$.

mechanical variations is that when $K_{m} / K<1$ transcritical flows can occur even when $\chi<\chi_{c e}$ and the transmural pressure is positive, i.e. $\alpha>1$ (e.g. the curve for $\chi=1$ in figure 7). Conversely, when $K_{m} / K>1$ transcritical flows can occur even when $\chi>\chi_{c e}$ and the transmural pressure is slightly positive, i.e. $\alpha>1$ (e.g. the curve for $\chi=65$ in figure 7).

If we consider an increase in cross-sectional area at the equilibrium, i.e. $A_{0 m} / A_{0}>1$, the transcritical curves move in the opposite direction, and critical conditions are more difficult to obtain. In general it is seen that for a given value of the stiffness parameter ratio $K_{m} / K$, a reduction of $A_{0 m}$ makes the occurrence of transcritical flows easier (either subcritical or supercritical), while an enlargement, say an increase in $A_{0 m}$, has the opposite effect.

\subsection{Complete tube law}

The complete tube law is obtained when $\beta_{1}>0$ and $\beta_{2}<2$. This relationship is used in a physiological context for describing the tube law for lungs (e.g. Elad et al. 1987) and veins (e.g. Brook, Falle \& Pedley 1999; Müller \& Toro 2013). In this case the critical condition in the modified region $\alpha_{m}=A_{c r} / A_{0 m}$ is obtained by imposing that $S_{m}=1$ in the definition (2.5) where $\chi=\chi_{m}$, which gives

$$
\alpha_{m}^{2}\left(\beta_{1} \alpha_{m}^{\beta_{1}}+\beta_{2} \alpha_{m}^{-\beta_{2}}\right)=\left[\chi \frac{K_{m}}{K}\left(\frac{A_{0 m}}{A_{0}}\right)^{2}\right]^{-1},
$$



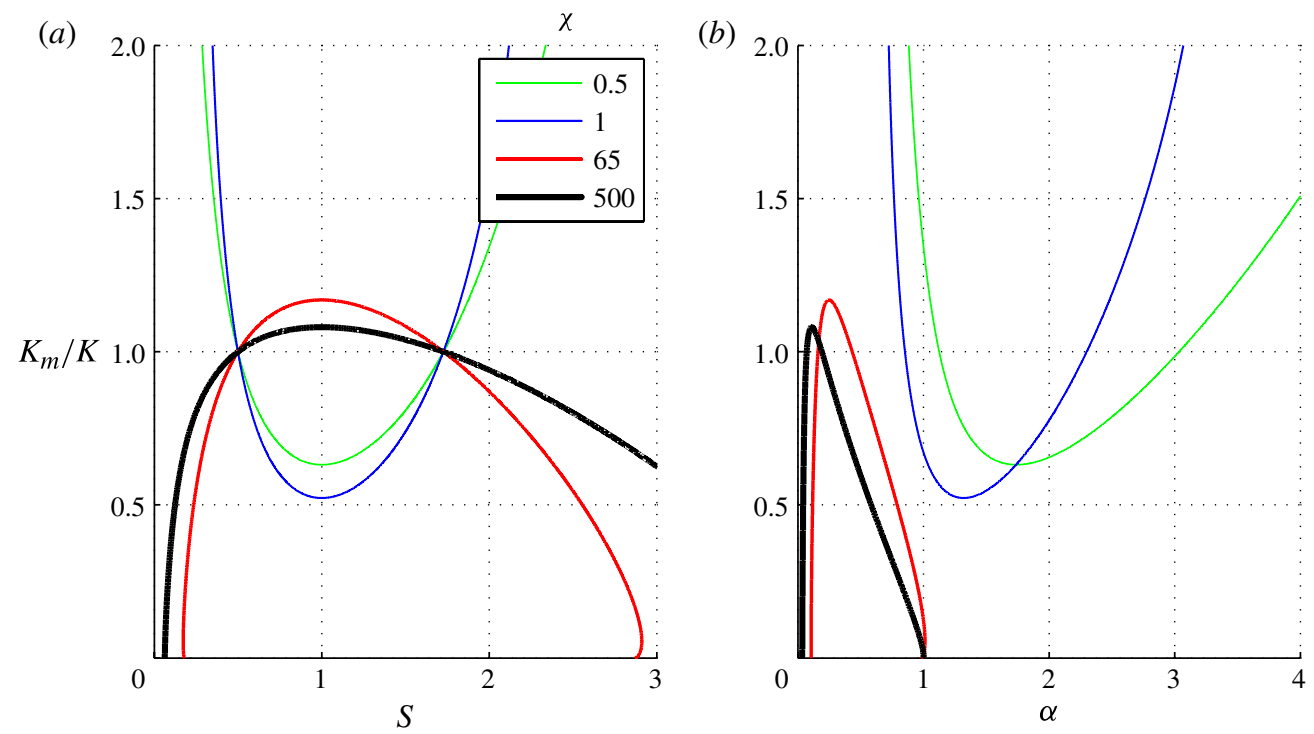

FIGURE 7. (Colour online) Geometrical and mechanical variations: transcritical curves $\left(S-K_{m} / K\right)(a)$ and $\left(\alpha-K_{m} / K\right)(b)$ for different values of $\chi$ when $A_{0 m} / A_{0}=0.75$. Results are obtained for the simplified tube law $\left(\beta_{1}=1 / 2, \beta_{2}=0, \chi_{c e}=2\right)$.

which does not admit algebraic solutions in closed form. Therefore, the strategy to determine the transcritical conditions is different from the case in $\S 3.1$. We rewrite the total pressure $\mathscr{P}$ from (2.11) as a function of $\alpha$. Then, we express $\mathscr{P}_{m}$ by subtracting the relationship $\mathrm{d} \mathscr{P} / \mathrm{d} \alpha=0$, which holds for critical conditions, in the modified region. Imposing $\mathscr{P}=\mathscr{P}_{m}$ finally yields the dimensionless relationship

$$
\frac{\mathscr{P}}{K}=\frac{1}{2 \chi \alpha^{2}}+\alpha^{\beta_{1}}-\alpha^{-\beta_{2}}=\frac{K_{m}}{K}\left[\left(\frac{\beta_{1}}{2}+1\right) \alpha_{m}^{\beta_{1}}+\left(\frac{\beta_{2}}{2}-1\right) \alpha_{m}^{-\beta_{2}}\right],
$$

where the first expression refers to the undisturbed conditions and the second one to the modified region. The transcritical curves can be obtained by means of the following procedure. First, (3.6) is numerically solved for the assigned value of $\chi$ and of the geometrical and mechanical changes. Then, the critical area ratio $\alpha_{m}$ is used in (3.7) to obtain the two corresponding values of $\alpha$. Finally, the limit values of the speed index are calculated going back to (2.5).

In this case, geometrical variations $\left(A_{0 m} / A_{0} \neq 1\right)$ without mechanical changes $\left(K_{m} / K=1\right)$ do not determine a unique curve in the $\left(S-A_{0 m} / A_{0}\right)$ plane (figure 8), as was the case for the simplified tube law (figure 4). However, similarly to that case, the transcritical region becomes narrower in the $\left(\alpha-A_{0 m} / A_{0}\right)$ plane for large values of $\chi>\chi_{c e}$, and shifts toward smaller values of $\alpha$ (negative transmural pressure). The shape of the curve is different, however, and a wider transcritical range of $\alpha$ remains for small values of $K_{m} / K$ with respect to the simplified tube law case.

An analogous correspondence is visible for the case of mechanical variations only $\left(K_{m} / K \neq 1, A_{0 m} / A_{0}=1\right.$, see figure 9$)$. In a similar way to the simplified tube law, there is a critical value $\chi_{c e}=\left(\beta_{1}+\beta_{2}\right)^{-1}$ for the existence of marginal curves for more/less rigid modified regions $\left(K_{m} / K \gtrless 1\right)$. For an increase in stiffness $\left(K_{m} / K>1\right)$ and $\chi<\chi_{c e}$, the transcritical curves are characterized by positive transmural pressures $(\alpha>1)$ (see figure $9 b)$; for the opposite case $\left(K_{m} / K<1, \chi>\chi_{c e}\right)$ transition can occur 

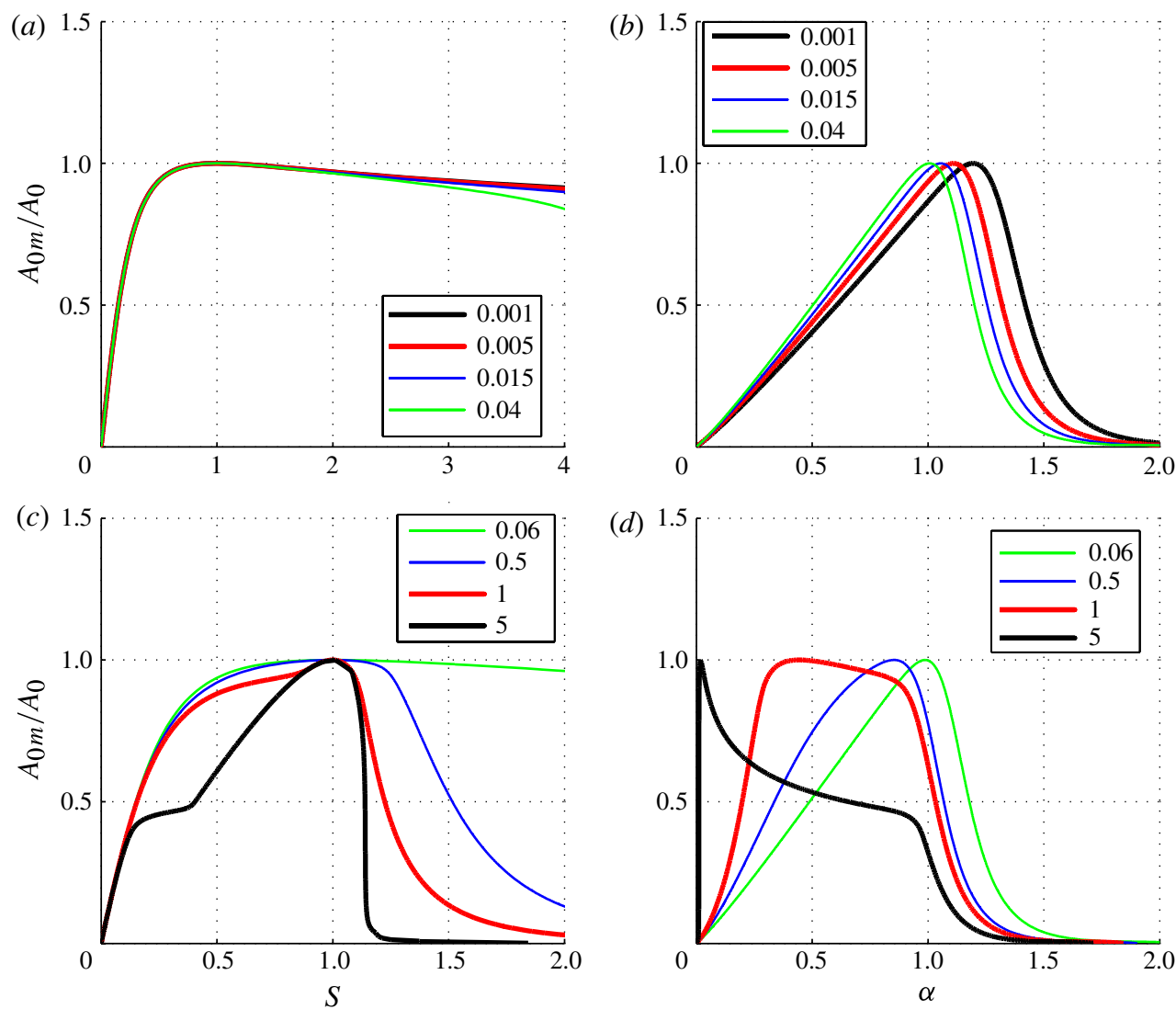

FIGURE 8. (Colour online) Geometrical variations for the complete tube law $\left(\beta_{1}=20\right.$, $\left.\beta_{2}=3 / 2, \chi_{c e}=0.0465\right)$ : transcritical conditions $A_{0 m} / A_{0}$ as a function of speed index $S(a, c)$ and area ratio $\alpha(b, d)$ for different values of $\chi$ (see legend) and for $K_{m} / K=1$.

only for negative values of the transmural pressure $(\alpha<1)$. It is worth noting that for very high values of $\chi$ (e.g. for very slow flows) the transcritical values of the area ratio $\alpha$ become lower and tend to prevent the occurrence of such a phenomenon, meaning that collapsible tubes are more likely to show transition at the maximum flow conditions than at rest.

In order to illustrate how a change in the mechanical properties can produce transcritical conditions, in figure 10 we analyse the steady profiles in a collapsible tube $\left(\beta_{1}=20, \beta_{2}=3 / 2\right)$ with a variation in the stiffness coefficient $K_{m}$. The solution has been obtained following the procedure described in the Appendix. The undisturbed flow, corresponding to an inviscid subcritical flow with along-stream uniform stiffness $K$, is characterized by a speed index $S=0.8$, and a value of the parameter $\chi=0.015<\chi_{c e}$. According to figure 9, the latter condition suggests that a transition can occur only for $K_{m}>K$. Results are represented in analogy with the panels in figures 3 and 5, the only difference being that now the mechanical properties change from $K$ to $K_{m}$ in the modified region (instead of the reference area $A_{0}$ ), as shown in figure $9(a)$. Two different configurations are analysed: the first in which the ratio $K_{m} / K=6$ is modified for a four-diameter length in the middle of the tube, and the second in which the same condition holds but for a ratio $K_{m} / K=7$. It 

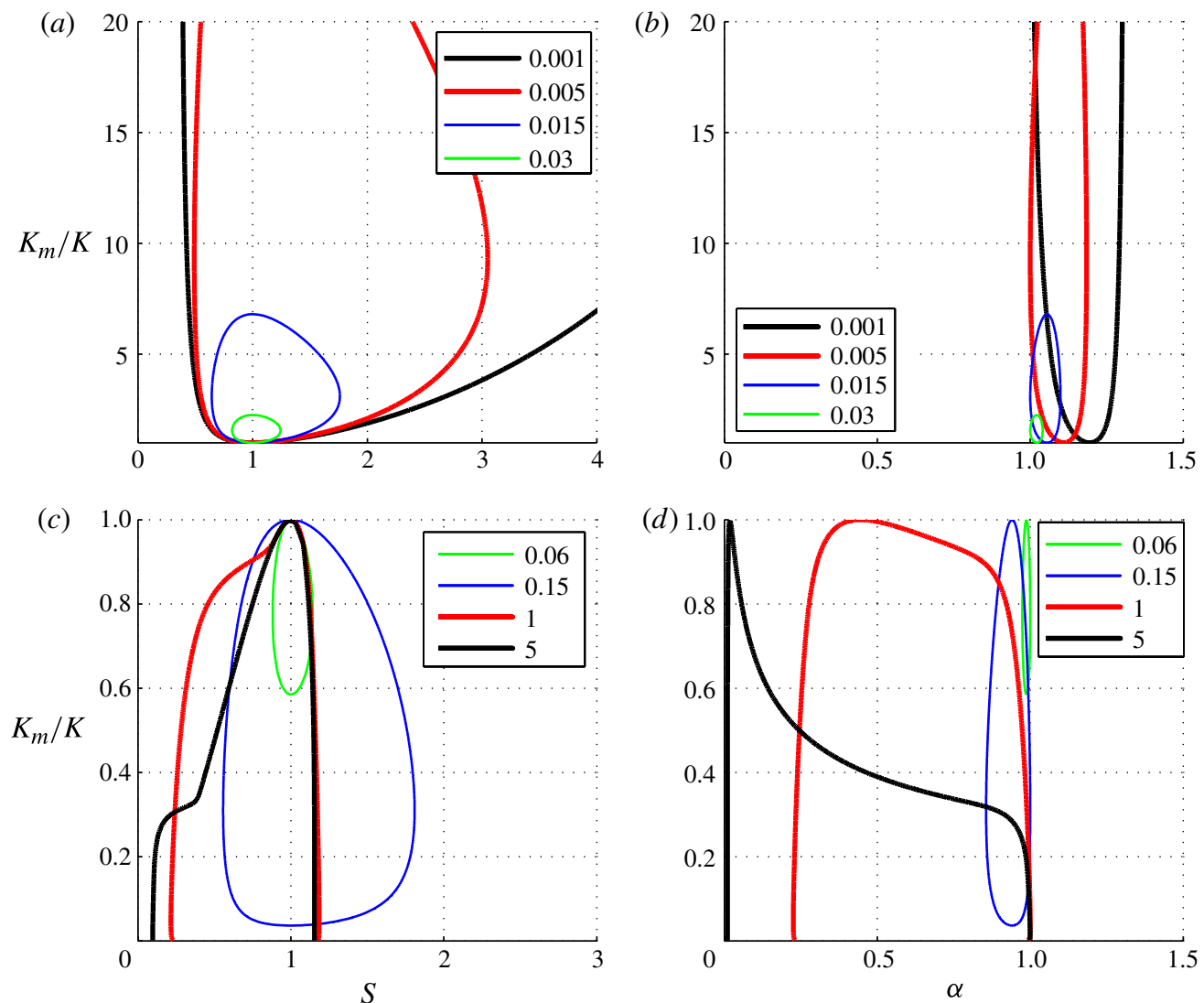

FIgURE 9. (Colour online) Mechanical variations for the complete tube law $\left(\beta_{1}=20\right.$, $\left.\beta_{2}=3 / 2, \chi_{c e}=0.0465\right)$ : transcritical conditions $K_{m} / K$ as a function of speed index $S(a, c)$ and area ratio $\alpha(b, d)$ for different values of $\chi$ (see legend) and for $A_{0 m} / A_{0}=1$.

is important to note that the transcritical region in figure 9 is closed, and in this analysis we consider the upper branch of the marginal curves, with a critical value of $K_{m} / K=6.3$ for the assigned value of the speed index. In particular, we expect to avoid transcritical flow for the largest increase in stiffness. This apparently counter-intuitive result is confirmed by the behaviour of the steady profiles in figure 10: for $K_{m} / K=7$ (dashed lines) the flow remains subcritical even if it reduces its cross-sectional area in the modified region, while for $K_{m} / K=6$ (solid lines) the flow passes through critical conditions and becomes supercritical. The speed index goes up to 1.36, but the increase in velocity is relatively low because of the limited decrease of the crosssectional area. A qualitatively different picture, with strong increase in velocity and decrease in area, would be noted in the lower branch of the marginal curve of the transcritical region for the same speed index but lower values of $K_{m} / K$.

\section{Implications of variation in mechanical properties for arteries and veins}

We can now discuss the relevance of the occurrence of transcritical flows to mechanical property variations in arteries and veins when a compliance mismatch exists. This case has a broad medical relevance since the presence of flow limitations 
(a)
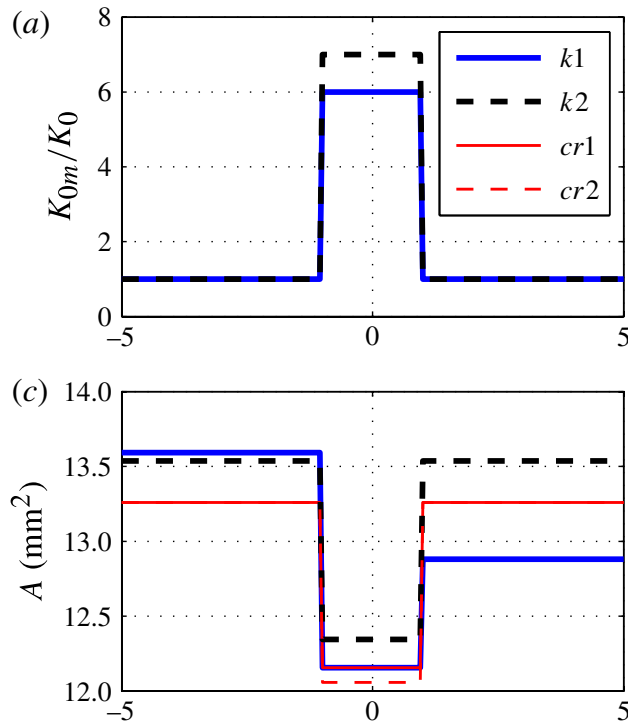

(e)

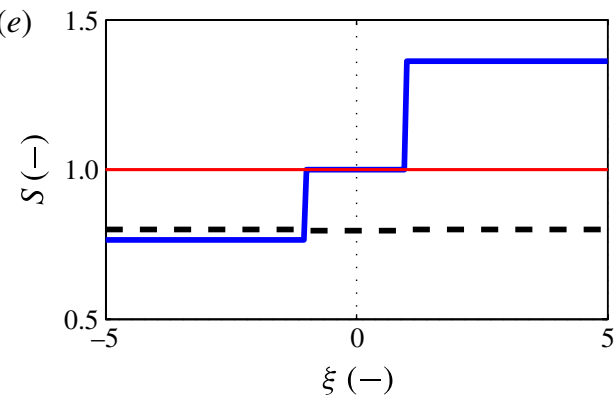

(b)

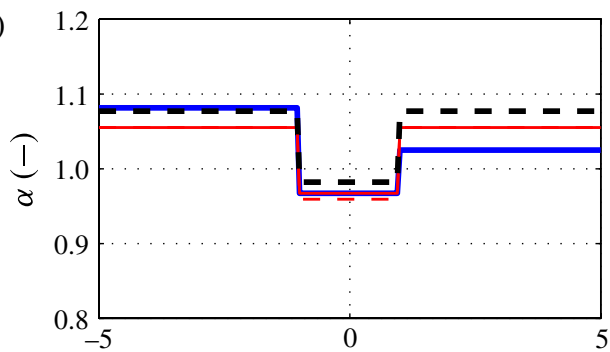

(d)
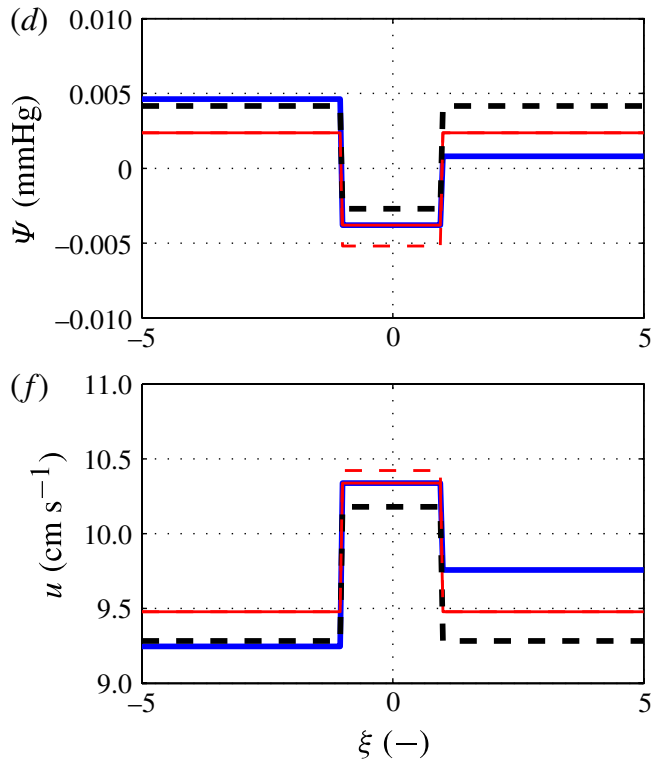

FIGURE 10. (Colour online) Steady profiles for an inviscid flow in a collapsible tube $(R=0$, $\left.\beta_{1}=20, \beta_{2}=3 / 2, \chi_{c e}=0.0465\right)$ with uniform reference area $\left(A_{0 m} / A_{0}=1\right)$ but discontinuous mechanical properties: no transition through critical condition occurs for $K_{m} / K=7$ (dashed black line, $k 2$ in the legend), while it does occur for the lower variation $K_{m} / K=6$ (solid blue line, $k 1$ in the legend). The reference undisturbed flow is subcritical $(S=0.8)$ with $\psi=4.17 \times 10^{-3} \mathrm{mmHg}\left(A=13.4 \mathrm{~mm}^{2}\right)$ as downstream boundary condition. The panels are as in figure 3, with the exception of $(a)$, where the ratio $K_{m} / K$ is shown. Other data: density $\rho=1050 \mathrm{~kg} \mathrm{~m}^{-3}$, discharge $Q=1.257 \mathrm{ml} \mathrm{s}^{-1}$, tube radius $R_{0}=0.2 \mathrm{~cm}\left(A_{0}=12.57 \mathrm{~mm}^{2}\right)$, stiffness $K=0.1575 \mathrm{~Pa}$, resulting in a value of the parameter $\chi=0.015$.

and elastic jumps can have adverse physiological effects. It occurs when prosthesis (grafts or stents) are surgically implanted for endovascular repairs. Another important medical aspect, which is not investigated here, is that transition through critical conditions could occur in large blood vessels because of an atherosclerotic plaque, which produces a stenosis. This has been investigated by $\mathrm{Ku}$ et al. (1990), who proposed a one-dimensional steady analysis, while the corresponding unsteady analysis is given in Downing \& $\mathrm{Ku}$ (1997).

\subsection{Arteries}

Let us consider as an example the following four arteries: the ascending, thoracic and abdominal aorta and the iliac artery. The data concerning the geometrical and mechanical properties of these vessels, collected from the literature, are given in 


$\begin{array}{lcllccc}\text { Artery } & E(\mathrm{kPa}) & h_{0}(\mathrm{~cm}) & R_{0}(\mathrm{~cm}) & K(\mathrm{~Pa}) & Q_{\max }\left(\mathrm{ml} \mathrm{s}^{-1}\right) & \chi(-) \\ \text { Ascending aorta } & 400 & 0.163 & 1.45 & 59954 & 470 & 112 \\ \text { Thoracic aorta } & 400 & 0.12 & 1.0 & 64000 & 303 & 65 \\ \text { Abdominal aorta } & 400 & 0.108 & 0.87 & 66206 & 123 & 235 \\ \text { Iliac artery } & 400 & 0.076 & 0.52 & 78948 & 38 & 370\end{array}$

TABLE 1. Mechanical and geometrical properties of different arteries: the Young's modulus of the vessel tissue $E$, the wall thickness $h_{0}$, and the radius of the vessel when the transmural pressure is set to zero $R_{0}$ have been extracted from Avolio (1980), while the maximum flow rate during the cardiac cycle $Q_{\max }$ has been collected from Reymond et al. (2009). $K$ is the stiffness parameter evaluated using relation (4.1).

table 1. The tube law used for describing the behaviour of arteries is described by relation (2.3), where the exponents are fixed to $\beta_{1}=1 / 2$ and $\beta_{2}=0$, while $K$ has been evaluated using the following classical formula derived by considering static equilibrium of the vessel wall and small vessel deformations (e.g. Sherwin et al. 2003; Formaggia, Quarteroni \& Veneziani 2009):

$$
K=\frac{\sqrt{\pi}}{\left(1-v^{2}\right)} \frac{E h_{0}}{\sqrt{A_{0}}},
$$

where $v$ is the Poisson's ratio set equal to 0.5. This simplified tube law correctly describes wave propagation patterns in arterial networks of arteries, as has been extensively confirmed by the existing literature (Avolio 1980; Liang et al. 2009; Alastruey et al. 2011). The parameter $\chi$ has been evaluated using relation (2.6), where $u_{0}=Q_{\max } / A_{0}$ and the blood density $\rho$ has been set to $1050 \mathrm{~kg} \mathrm{~m}^{-3}$.

It is seen that, for the arteries taken into consideration, the parameter $\chi$ ranges between 65 and 370. These can be considered the minimum values that can occur since the flow rate can vanish during the cardiac cycle and $\chi$ goes to infinity. It is also worth remarking that, despite the fact that systematic experiments with the aim of estimating $\chi$ are not yet available, and that the $\chi$ data contained in table 1 have been obtained using what is currently available in the literature, the order of magnitude of the minimum value of this parameter is reliable, i.e. $O\left(10^{1}-10^{2}\right)$.

Since the parameter $\chi$ typical of arteries (see table 1) is greater than $\chi_{c e}=2$, transition through critical conditions can occur only if $K_{m} / K<1$ (i.e. a reduction in the vessel's stiffness: see figure $6 c, d$ ). The maximum values of the speed index typical of arteries ranges between 0.2 and 0.25 (Caro et al. 2012), which implies that transition through the critical conditions can occur provided that $K_{m} / K \lesssim 0.4$ (see figure $6 c$ ) and $\alpha<1$ (see figure $6 d$ ). From this second condition it follows that critical conditions occur if the transmural pressure is negative, which, for arteries, is a condition that physiologically does not apply. Moreover, the limiting condition $\alpha<1$ continues to hold even when the cross-sectional area of the implanted graft/stent reduces the lumen by $25 \%$ (see figure 7) or it is increased. We can conclude that, in arteries, variations of mechanical properties seem highly unlikely to induce transcritical conditions, i.e. they are not able to change the subcritical status of the incoming flow. 


$\begin{array}{lcccccc}\text { Vein } & E(\mathrm{kPa}) & h_{0} / R_{0} & K(\mathrm{~Pa}) & u\left(\mathrm{~cm} \mathrm{~s}^{-1}\right) & \chi(-) & \text { Source } \\ \text { Saphenous vein } & 27 & 0.029 & 0.069 & 7 & 0.013 & (\mathrm{We}, \mathrm{Ab}) \\ \text { Jugular vein } & 100 & 0.035 & 0.47 & 30 & 0.019 & \text { (At, St) } \\ \text { Vena cava } & 260 & 0.05 & 3.61 & 15 & 0.152 & \text { (Ni) }\end{array}$

TABLE 2. Mechanical and geometrical properties of different veins: $u$ is a fluid velocity measured inside the vessel, $K$ is the stiffness parameter evaluated using relation (4.2). The acronyms refer to the publication from which specific data were extracted: We, Wesly et al. (1975); Ab, Abraham et al. (1994); At, Attinger (1969); St, Stoquart-ElSankari et al. (2009); Ni, Nippa, Alexander \& Folse (1971).

\subsection{Veins}

In order to analyse the possible occurrence of transcritical flows in veins we consider the following three blood vessels: the saphenous vein, the jugular vein and the vena cava. Geometrical and mechanical properties of such vessels are given in table 2.

Some experimental studies have demonstrated that the structural performance of veins is similar to that of a thin-walled elastic tube (Drzawiecki et al. 1997; Bassez, Flaud \& Chauveau 2001). Then, the relationship that locally correlates between the transmural pressure and the vein's cross-sectional area can be reasonably described by the complete tube law (2.3), where the exponent $\beta_{2}$ is set to $3 / 2$ (Shapiro 1977; Brook et al. 1999), which well describes the collapse of the thin wall, and the exponent $\beta_{1}$ is chosen equal to 20, so that for values of $\alpha<0$ the tube law behaves like $\psi=-K \alpha^{-3 / 2}$ (Flaherty, Keller \& Rubinow 1972). The stiffness parameter, corresponding to the bending stiffness of a thin walled tube (Brook et al. 1999; Müller \& Toro 2013) is assumed to be equal to

$$
K=\frac{E}{12\left(1-v^{2}\right)}\left(\frac{h_{0}}{R_{0}}\right)^{3} .
$$

The parameter $\chi$ has been evaluated using relation (2.6), where $u_{0}$ has been approximated using available measures of the flow velocity $u$ (see table 2), and $\rho$ is the same as for arteries. In this case the order of magnitude of the parameter $\chi$ is $O\left(10^{-1}-10^{-2}\right)$ and the same conclusions drawn for arteries, concerning the reliability of these data, can be applied here as well.

As emerges from table 2, the parameter $\chi$ for veins can be higher or smaller than $\chi_{c e}=0.05$, which means that transcritical flows can occur for values $K_{m} / K \lessgtr 1$ (see figure 9), i.e. both stiffer and softer endovascular repairs can potentially induce transition. If we consider veins for which $\chi \simeq 0.15$, transition can occur if the incoming flow is subcritical and $\alpha$ is slightly smaller than one (moderate negative transmural pressure). If we consider veins characterized by values of $\chi$ which are one order of magnitude smaller, e.g. $\chi \simeq 0.015$, from figure $9(c, d)$ we can gather that transition can occur when the speed index is higher than $\sim 0.4$ and $\alpha$ is slightly higher than one (small but positive transmural pressure). Since the transmural pressure in veins is smaller than that in arteries and can achieve moderately negative values, and the speed index can be higher than those for arteries, we can conclude that in veins is likely that abrupt variations of the stiffness parameter $K$ may induce transitions through critical conditions. Finally, it is worth mentioning that the same conclusion can be drawn if smaller (i.e. $\beta_{1}=10$ ) or higher (i.e. $\beta_{1}=30$ ) values for the coefficient $\beta_{1}$ are considered. 


\section{Conclusions}

We have performed a steady and inviscid analysis and found transcritical curves that describe the conditions under which the incoming flow inside a collapsible tube may pass through the critical state due to an abrupt change of the mechanical properties of the wall vessel. Such marginal curves are parametric and are a function of the dimensionless number $\chi=K /\left(\rho u_{0}^{2}\right)$. We investigated two different cases. In the first case we assumed a simplified tube law (i.e. $\beta_{2}=0$ ), and in the second we considered a complete tube law (i.e. $\beta_{1} \geqslant 0$ and $0<\beta_{2} \leqslant 2$ ). For both cases we described the full range of conditions for which transition can occur. In particular we identified the values of the speed indexes, one supercritical and one subcritical, which are the limits of the transcritical region, and the associated values of the area ratio $\alpha$, which are related to the values of the transmural pressure.

Moreover, we analysed the situation of medical interest in which prostheses are implanted in arteries and veins in order to repair vessel diseases. We found that in arteries, the presence of a mismatch in the Young's modulus is highly unlikely to produce transition through critical conditions, while, on the other hand, the transition is likely to occur in veins. Of course, since physiological blood flow in arteries and veins is actually unsteady, three-dimensional and viscous, further refinements are required for a detailed analysis in real medical cases. Finally, we believe that this analysis can represent a preliminary tool for the identification of possible risks related to interventions that modify the geometrical or mechanical properties of biological collapsible tubes.

\section{Appendix}

In this appendix we describe how to obtain numerically steady-state profiles of flows in collapsible tubes.

Equation (2.9) with (2.8) can be manipulated so as to obtain the following expression for the area variation:

$$
\frac{\mathrm{d} A}{\mathrm{~d} x}=\frac{\frac{A}{\rho} \frac{\mathrm{d} p_{e}}{\mathrm{~d} x}-\frac{\mathrm{d} \psi}{\mathrm{d} \alpha} \frac{1}{\rho}\left(\frac{A}{A_{0}}\right)^{2} \frac{\mathrm{d} A_{0}}{\mathrm{~d} x}+\frac{A \psi}{K \rho} \frac{\mathrm{d} K}{\mathrm{~d} x}+R u}{\frac{\mathrm{d} \psi}{\mathrm{d} \alpha} \frac{A}{\rho A_{0}}\left(S^{2}-1\right)} .
$$

Following the classical Poiseuille solution for laminar flows, we estimate

$$
R=8 \pi \mu / \rho,
$$

where $\mu$ is the fluid viscosity, assumed to be equal to $4.0 \times 10^{-3} \mathrm{~Pa}$ s for the simulations in this paper.

This equation governs the steady-state behaviour of a flow inside a collapsible tube and describes the behaviour of $A$ as function of the spatial variations of the stiffness parameter $K$, the cross-sectional area at equilibrium $A_{0}$ and the external pressure $p_{e}$. Steady solutions can be obtained solving (A 1) directly using any solver for ordinary differential equations. One of the major problems of this method of solution is related to the fact that a singularity appears for $S=1$ in (A 1).

In this work we implement a slightly different technique which overcomes the problem mentioned above. Specifically, we take advantage of what is usually done for the solution of open channel flows in river hydraulics. We impose the condition that the total pressure (2.11) obeys the following relationship between two sections (up 
indicating upstream, down downstream) of a distance of length $\Delta x$ :

$$
\mathscr{P}_{\text {down }}=\mathscr{P}_{u p}-\rho \int_{\Delta x} \frac{R u}{A} \mathrm{~d} x .
$$

This implies that in the inviscid case $\mathscr{P}$ does not vary. Thus, we start from the upstream boundary condition (expressed in terms of area or, equivalently, transmural pressure), and we proceed downstream looking only for the supercritical values $A_{\text {sup }}$. If the solution in the downstream cross-sectional area does not exist, we impose the critical value $A_{c r}$. Similarly, we determine the subcritical profile $A_{s u b}$ starting from the downstream boundary condition (imposed as $A$ or $\psi$ in this case as well) and proceeding upstream. Having simultaneously determined two profiles (super- and subcritical), we need to choose the correct values of the area in each cross-section. The physically admissible solution is the profile that is consistent with the requirement (A 3) along the profile, suggesting that a smooth transition can occur only through the critical conditions where the two profiles have the same (minimum) value of $\mathscr{P}$. On the other hand, the abrupt transition across an elastic jump is solved selecting the profile characterized by the highest momentum flux, i.e. the maximum value between $\mathscr{M}\left(A_{\text {sub }}\right)$ and $\mathscr{M}\left(A_{\text {sup }}\right)$ where

$$
\begin{aligned}
\mathscr{M}(A) & =\rho \frac{Q^{2}}{A}+A\left(p_{e}+\psi\right)-\int_{A_{0}}^{A} \psi \mathrm{d} A \\
& =\rho \frac{Q^{2}}{A}+A p_{e}+K A\left[\frac{\beta_{1}}{1+\beta_{1}}\left(\alpha^{\beta_{1}}-1\right)+\frac{\beta_{2}}{1-\beta_{2}}\left(\alpha^{-\beta_{2}}-1\right)\right] .
\end{aligned}
$$

It is worth mentioning that the minimum value of $\mathscr{M}$, obtained by imposing that $\left.(\mathrm{d} \mathscr{M} / \mathrm{d} A)\right|_{Q, K, A_{0}}=0$, is found for $A=A_{c r}$. Then both functions $\mathscr{P}(A)$ and $\mathscr{M}(A)$ have the same minimum value.

\section{REFERENCES}

Abraham, P., Leftheriotis, G., Desvaux, B., Saumet, M. \& Saumet, J. L. 1994 Diameter and blood velocity changes in the saphenous vein during thermal stress. Eur. J. Appl. Physiol. 69, 305-308.

Alastruey, J., Khir, A. W., Matthys, K. S., Segers, P., Sherwin, S. J., Verdonck, P. R., PARKer, K. H. \& Peiró, J. 2011 Pulse wave propagation in a model human arterial network: assessment of 1-D visco-elastic simulations against in vitro measurements. J. Biomech. 44, 2250-2258.

Alastruey, J., Parker, K. H., Peiró, J., Byrd, S. M. \& Sherwin, S. J. 2007 Modelling the circle of Willis to assess the effects of anatomical variations and occlusions on cerebral flows. J. Biomech. 40, 1794-1805.

AtTinger, E. O. 1969 Wall properties of veins. IEEE Trans. Biomed. Engng 16, 253-261.

Avolio, P. 1980 Multi-branched model of the human arterial system. Med. Biol. Engng Comput. 18, 709-718.

Bassez, S., Flaud, P. \& Chauveau, M. 2001 Modelling of the deformation of flexible tubes using a single law: application to veins of the lower limb in man. Trans. ASME: J. Biomech. Engng 123, 58-65.

Brook, B. S., Falle, S. A. E. G. \& Pedley, T. J. 1999 Numerical solutions for unsteady gravity-driven flows in collapsible tubes: evolution and roll-wave instability of a steady state. J. Fluid Mech. 396, 223-256.

Cancelli, C. \& Pedley, T. J. 1985 A separated-flow model for collapsible-tube oscillations. J. Fluid Mech. 157, 375-404.

CANIĆ, S. 2002 Blood flow through compliant vessels after endovascular repair: wall deformations induced by discontinuous wall properties. Comput. Vis. Sci. 4, 147-155. 
Canić, S., Ravi-Chandar, K., Krajcer, Z., Mirkovic, D. \& Lapin, S. 2005 Mathematical model analysis of Wallstent ${ }^{\circledR}$ and AneuRx ${ }^{\circledR}$ : dynamic responses of bare-metal endoprosthesis compared with those of stent-graft. Texas Heart Institute J. 32 (4), 502-506.

Caro, C. G., Pedley, T. J., Schroter, R. C. \& Seed, W. A. 2012 The Mechanics of the Circulation, 2nd edn. Cambridge.

Charonko, J. J., Ragab, S. A. \& Vlachos, P. P. 2009 A scaling parameter for predicting pressure wave reflection in stented arteries. J. Med. Dev. 3, 011006.

Downing, J. M. \& KU, D. N. 1997 Effects of frictional losses and pulsatile flow on the collapse of stenotic arteries. Trans. ASME: J. Biomech. Engng 119, 317-324.

Drzawiecki, G., Field, S., Moubarak, I. \& Li, J. 1997 Vessel growth and collapsible pressure area relationship. Am. J. Physiol. Heart Circ. Physiol. 273, H2030-H2043.

ELAD, D. \& KAMM, R. D. 1989 Parametric evaluation of forced expiration using a numerical model. Trans. ASME: J. Biomech. Engng 111, 192-199.

Elad, D., Kamm, R. D. \& Shapiro, A. H. 1987 Chocking phenomena in a lung-like model. Trans. ASME: J. Biomech. Engng 109, 1-92.

Elad, D., KAMM, R. D. \& Shapiro, A. H. 1988 Mathematical simulation of forced expiration. J. Appl. Physiol. 65, 14-25.

Elad, D., KAmm, R. D. \& Shapiro, A. H. 1989 Steady compressible flow in collapsible tubes: application to forced expiration. J. Fluid Mech. 203, 401-418.

Flaherty, J. E., Keller, J. B. \& Rubinow, S. I. 1972 Post buckling behavior of elastic tubes and rings with opposite sides in contact. SIAM J. Appl. Maths 23, 446-455.

Formaggia, L., Quarteroni, A. \& Veneziani, A. 2009 Cardiovascular Mathematics: Modelling and Simulation of the Circulatory System. Springer.

Grotberg, J. B. \& Jensen, O. E. 2004 Biofluid mechanics in flexible tubes. Annu. Rev. Fluid Mech. 36, 121-147.

Heil, M. \& Hazel, A. L. 2011 Fluid-structure interaction in internal physiological flows. Annu. Rev. Fluid Mech. 43, 141-162.

Jensen, O. E. \& Pedley, T. J. 1989 The existence of steady flow in a collapsed tube. J. Fluid Mech. 206, 339-374.

Kadoglou, N. P. E., Moulakakis, K. G., Papadakis, I., Ikonomidis, I., Alepaki, M., LEKAKIS, J. \& LiAPIS, C. D. 2012 Changes in aortic pulse wave velocity of patients undergoing endovascular repair of abdominal aortic aneurysms. J. Endovasc. Ther. 19, 661-666.

Ku, D. N., Zeigler, M. N. \& Downing, J. M. 1990 One-dimensional steady inviscid flow through a stenotic collapsible tube. Trans. ASME: J. Biomech. Engng 112, 444-450.

Lantelme, P., Dzudie, A., Milon, H., BriccA, G., Legedz, L., Chevalier, J. M. \& FEUGIER, P. 2009 Effect of abdominal aortic grafts on aortic stiffness and central hemodynamics. J. Hypertension 27, 1268-1276.

Liang, F. Y., TAKagi, S., Himeno, R. \& LiU, H. 2009 Biomechanical characterization of ventricular-arterial coupling during aging: a multi-scale model study. J. Biomech. 42, 692-704.

MARCHI, E. 1968 Il calcolo del rigurgito provocato dalle pile dei ponti: deflusso con transizione attraverso lo stato critico. L'Energia Elettrica 4, 254-259.

Müller, L. O., PARÉs, C. \& TORO, E. F. 2013 Well-balanced high-order numerical schemes for one-dimensional blood flow in vessels with varying mechanical properties. J. Comput. Phys. 242, 53-85.

Müller, L. O. \& Toro, E. F. 2013 A global multi-scale mathematical model for the human circulation with emphasis on the venous system. Preprint NI3007, Isaac Newton Institute for Mathematical Sciences.

Nippa, J. H., Alexander, R. H. \& Folse, R. 1971 Pulse wave velocity in human veins. J. Appl. Physiol. 30 (4), 558-563.

Pedley, T. J. 2000 Blood flow in arteries and veins. In Perspective in Fluid Dynamics (ed. G. K. Batchelor, H. K. Moffat \& M. G. Worster), Cambridge.

Pedley, T. J., Brook, B. S. \& Seymour, R. S. 1996 Blood pressure and flow rate in the giraffe jugular vein. Phil. Trans. R. Soc. Lond. B 351, 855-866. 
Reymond, P., Merenda, F., RÜfenacht, D. \& Stergiopulos, N. 2009 Validation of a one-dimensional model of the systemic arterial tree. Am. J. Physiol. Heart Circ. Physiol. 297, 208-222.

Sakalihasan, N., Limet, R. \& Defawe, O. D. 2005 Abdominal aortic aneurysm. Lancet 365 (9470), 1577-1589.

Shapiro, A. H. 1977 Steady flow in collapsible tubes. Trans. ASME: J. Biomech. Engng 99, $126-147$.

Sherwin, S. J., Formaggia, L., Peiro, J. \& Franke, V. 2003 Computational modelling of 1D blood flow with variable mechanical properties and its application to the simulation of wave propagation in the human arterial system. Intl J. Numer. Meth. Fluids 43 (6-7), 673-700.

Stoquart-ElSankari, S., Lehmann, P., Villette, A., Czosnyka, M., Meyer, M. E., DERAMOND, H. \& BALÉDENT, O. 2009 A phase-contrast MRI study of physiologic cerebral venous flow. J. Cerebr. Blood F. Met. 29, 1208-1215.

TORO, E. F. \& Siviglia, A. 2013 Flow in collapsible tubes with discontinuous mechanical properties: mathematical model and exact solutions. Commun. Comput. Phys. 13 (2), 361-385.

Vardoulis, O., Coppens, E., Martin, B., Reymond, P., Tozzi, P. \& Stergiopulos, N. 2011 Impact of aortic grafts on arterial pressure. Eur. J. Vasc. Endovasc. Surg. 42, 704-710.

Wesly, R. L., Vaishnav, R. N, Fuchs, J. C, Patel, D. J. \& Greenfield, J. C. 1975 static linear and nonlinear elastic properties of normal and arterialized venous tissue in dog and man. Circulat. Res 37, 509-520.

Zamboni, P., Galeotti, R., Menegatti, E, Malagoni, A. M., Gianesini, S., Bartolomei, I., MASCOLI, F. \& SAlvi, F. 2009 A prospective open-label study of endovascular treatment of chronic cerebrospinal venous insufficiency. J. Vascu. Surg. 50, 1348-1358. 